\title{
Damping Control in Power Systems under Constrained Communication Bandwidth: A Predictor Corrector Strategy
}

\author{
Nilanjan Ray Chaudhuri, Member, IEEE, Debraj Chakraborty, Member, IEEE \\ and Balarko Chaudhuri, Member, IEEE
}

\begin{abstract}
Damping electromechanical oscillations in power systems using feedback signals from remote sensors is likely to be affected by occasional low bandwidth availability due to increasing use of shared communication in future. In this paper, a predictor corrector (PC) strategy is applied to deal with situations of low feedback data rate (bandwidth) where conventional feedback would suffer. Knowledge of nominal system dynamics is used to approximate (predict) the actual system behavior during intervals when data from remote sensors are not available. Recent samples of the states from a reduced observer at the remote location are used to periodically reset (correct) the nominal dynamics. The closed-loop performance deteriorates as the actual operating condition drifts away from the nominal dynamics. Nonetheless, significantly better performance compared to conventional feedback is obtained under low bandwidth situations. The analytical criterion for closed-loop stability of the overall system is validated through a simulation study. It is demonstrated that even for reasonably low data rates the closed-loop stability is usually ensured for a typical power system application confirming the effectiveness of this approach. The deterioration in performance is also quantified in terms of the difference between the nominal and off-nominal dynamics.
\end{abstract}

Index Terms-Damping Control, Power Systems, Electromechanical oscillation, Observer, Data Feedback Rate, Statefeedback, Predictor Corrector

\section{LiST OF NOTATIONS}

$G_{n} \quad$ Reduced order state space model of power system at nominal condition

$G_{i} \quad$ Reduced order state space model of power system at $i^{\text {th }}$ off-nominal condition

$L \quad$ Observer gain vector

$K \quad$ State feedback gain vector

$\sigma \quad$ Time interval between consecutive samples arriving at control center

$x_{i} \quad$ State vector of reduced power system model under $i^{t h}$ off-nominal condition

$\bar{x} \quad$ State vector estimated by the observer

$x_{n} \quad$ State vector of $G_{n}$

Support from the EPSRC, UK under grant EESC P11121 and Royal Academy of Engineering under Researcher Exchange Program in gratefully acknowledged.

N. Chaudhuri and B. Chaudhuri are with the Control and Power Research group, Imperial College London, London, UK (e-mail: n.chaudhuri@imperial.ac.uk,b.chaudhuri@imperial.ac.uk).

D. Chakraborty is with Indian Institute of Technology Bombay, Mumbai, India (e-mail: dc@ee.iitb.ac.in).

\author{
$t_{k} \quad$ Time instant of state resetting in $G_{n}$ \\ $x_{k} \quad$ Observer estimated state at time $t_{k}$ \\ $x_{k}^{\prime} \quad$ Actual state of reduced power system \\ model at time $t_{k}$ \\ $u(t) \quad$ Control input to the actuator \\ $\bar{u}(t) \quad$ Control input calculated at the sensor location \\ $\tilde{A}, \tilde{B}, \tilde{C}$ Deviation in actual operating condition \\ from nominal \\ $e(t) \quad$ Error between observer and estimated \\ (by $G_{n}$ ) states \\ $E(t) \quad$ Error between estimated (by $G_{n}$ ) \\ and actual states of reduced model \\ $\|\cdot\| \quad$ Euclidian norm of a vector or a matrix \\ $t^{*} \quad$ Time instant when $\|E(t)\|$ is maximum
}

\section{INTRODUCTION}

$\mathbf{F}$ EEDBACK data rate is often limited by the available bandwidth of communication channels and could be critical for satisfactory closed-loop performance. Especially, for networked control systems relying on communication of feedback signals from distant sensors, bandwidth limitation is a matter of serious concern. In the past researchers have focussed on desirable properties of communication networks to guarantee a minimum performance level with conventional control approach [1], [2], [3]. Also a lot of attention has been devoted to assessing the stability and performance of controllers connected over standard communication networks [4], [5], [6].

A novel control architecture was proposed in [7], [8] which can produce satisfactory performance up to an extent even with very low feedback data rates. The basic idea is to exploit the knowledge of nominal system dynamics to approximate (predict) the actual system behavior during intervals when data from remote sensors are not available. Recent samples of the states estimated from a reduced observer at the remote location are used to periodically reset (correct) the nominal dynamics. Throughout the rest of this paper, this idea would be referred to as predictor corrector (PC) approach. With such a strategy satisfactory closed-loop performance could be ensured up to a certain data rate depending on the difference between the nominal and actual system dynamics for both linear [7], [9] and nonlinear [10] systems.

In this paper, the above concept is applied in the context of power systems to damp low frequency electromechanical 
oscillations resulting out of generators in one geographical area swinging with respect to others in different regions [11]. Feedback signals from sensors (Phasor Measurement Units (PMUs)) located over diverse geographical areas are considered for higher observability - an approach commonly known in power systems literature as wide-area damping control (WADC). Effectiveness of WADC employing GPS synchronized measurements from remote sensors is well reported in power systems literature [12], [13], [14], [15]. Conventional feedback $(\mathrm{CF})$ control is used for WADC wherein the remote measurements (e.g. magnitude/phase angles of voltage, current) are transmitted via communication link to distant control centers. One of the concerns, however, is the adverse impact of data communication problems like latency, low data feedback rate etc. on the closed-loop performance and hence secure operation of power systems. This in fact has inhibited practical deployment of WADC till today except for a few prototype or pilot schemes.

In most of the present day installations dedicated communication infrastructure is used for power systems applications like online monitoring and discrete controls including special protection schemes [16], [17], [18]. Bandwidth is not a problem with such dedicated links and data rates are limited to about 25-60 samples per second mainly by the sampling rate of the sensors [19]. For controlling low frequency ( 0.1 to 2.0 $\mathrm{Hz}$ ) electromechanical oscillations above data rates are more than adequate. However, with future smart electricity grids relying more and more on communication the utilities are contemplating increasing use of shared instead of dedicated links. Only a part of the available bandwidth might be available for WADC sharing the rest between other data intensive services like substation networking [20] and even broadband communication [21]. A recent paper on latency computation for a hypothetical WADC in the context of Western Electricity Coordinating Council (WECC) system conjectured a hierarchical configuration of data communication [22]. Possible use of a shared communication was indicated with a large number of signals from diverse geographical locations communicated to many distant zonal phasor data concentrators (PDCs) [22].

With shared communication likely to be more common for power systems applications in future, our objective here is to demonstrate the application of a predictor corrector (PC) approach that is capable of producing satisfactory closed-loop performance despite occasionally unavoidable low feedback data rates. With the PC strategy, satisfactory closed-loop performance is achieved even with $1 \mathrm{~Hz}$ while performance with conventional feedback (CF) deteriorates significantly below a data rate of $10 \mathrm{~Hz}$. It should be mentioned that with normal data rates $(25-60 \mathrm{~Hz}) \mathrm{CF}$ could be used while switching to $\mathrm{PC}$ strategy below a certain threshold indicated by the time-stamp information at either end [23].

The basic philosophy behind the PC approach is to to exploit the knowledge of nominal system dynamics during the intersampling time interval. Hence its performance would depend to a large extent on the difference between the actual operating condition and the nominal dynamics. The deterioration in performance is quantified here in terms of the difference between the linearized systems at nominal and off-nominal operating conditions. Case studies are carried out on a test system under several operating scenarios to compare the performance with PC strategy against CF control for different feedback data rates. Despite the deterioration under off-nominal conditions, PC approach produces significantly better performance than $\mathrm{CF}$ with low data rates.

The overall stability of the closed-loop system depends on the length of inter-sampling time interval which is decided by the data rate. Below a certain data rate, derived analytically in [8], [10], the system would be unstable depending on the difference between nominal and actual operating conditions. Here the stability limit in terms of the minimum allowable data rate is verified through case studies across different scenarios. It is demonstrated that even for reasonably low data rates (e.g. $0.3-0.5 \mathrm{~Hz}$ ) the closed-loop stability is ensured confirming the effectiveness of the PC approach.

The main contributions of this paper are:

- Application of a predictor corrector (PC) strategy for damping electromechanical oscillations in power systems to achieve satisfactory dynamic response with low feedback data rates

- Analyze the inter-sampling error in terms of difference between the nominal (used to predict and correct) and off-nominal dynamics

- Compare the performance of PC against conventional feedback (CF) for different feedback data rates across different scenarios

- Validate the analytical stability limit with respect to minimum permissible feedback data rate against simulation results across different scenarios

The rest of the paper is organized as follows. Following this introductory section, the principles of the PC strategy is described in Section II. Assessment of overall stability in terms of minimum allowable data rate is discussed in section III. Quantification of performance deterioration under offnominal conditions is presented in Sections IV and V. A case study on a 4-machine, 2-area test system and a larger (16machine, 5-area) one is presented in Section VI to illustrate the effectiveness of the PC approach under low data rate situation.

\section{Predictor Corrector (PC) Strategy}

The predictor corrector (PC) strategy exploits the knowledge of the nominal system dynamics to predict the actual system behavior between two consecutive data samples which is corrected every time fresh estimates of states are available from the sensor location [8], [7]. At each instant the states of the reduced order nominal system model $\left(G_{n}\right)$ at the actuator location are calculated (predicted) with periodic resetting (corrected) at a lower rate (depending on feedback data rate) with the most recent states estimated by an observer at the sensor location as shown in Fig. 1.

The reduced order linearized model of the power system around the nominal operating condition $G_{n}$ is given by:

$$
G_{n}=\left[\begin{array}{c|c}
A_{n} & B_{n} \\
\hline C_{n} & 0
\end{array}\right]
$$

where, $A_{n} \in \Re^{m \times m}, B_{n} \in \Re^{m \times n}$ and $C_{n} \in \Re^{p \times m}$. Reduced order linearized model about the $i^{\text {th }}$ operating condition $G_{i}$ 


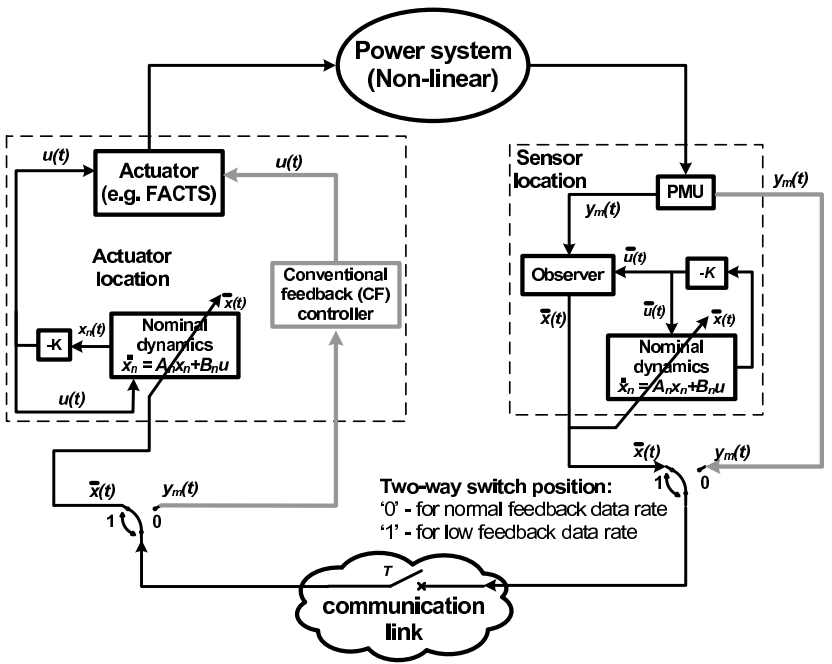

Fig. 1. Overall architecture including conventional feedback (CF) (in grey) and predictor corrector (PC) approach

(e.g. line outage, larger power transfer) is denoted as:

$$
G_{i}=\left[\begin{array}{c|c}
A_{n}+\tilde{A} & B_{n}+\tilde{B} \\
\hline C_{n}+\tilde{C} & 0
\end{array}\right]
$$

where, $\tilde{A}, \tilde{B}, \tilde{C}$ represent the deviation around the nominal operating condition and $A_{i}=A_{n}+\tilde{A}, B_{i}=B_{n}+\tilde{B}, C_{i}=$ $C_{n}+\tilde{C}, D_{i}=0$. The states of $G_{n}$ and $G_{i}$ are denoted as $x_{n}(t)$ and $x_{i}(t)$, respectively. Exogenous disturbances could also be incorporated in (2), if required.

An observer (3) at the distant sensor location estimates the states $\bar{x}$ of the reduced order system which are transmitted through the communication network to the controller. The state equation of the observer is:

$$
\dot{\bar{x}}=\left(A_{n}-L C_{n}\right) \bar{x}+B_{n} \bar{u}+L C_{i} x_{i}
$$

Note that the observer at the PMU location requires knowledge of control input $u(t)$ which is calculated $(\bar{u}(t))$ using the reduced-order model $G_{n}$ and the state-feedback gain vector $K$, see Fig. 1. This nominal dynamics at the actuator location can be described by the following equation:

$$
\dot{x}_{n}(t)=A_{n} x_{n}(t)+B_{n} u(t)
$$

Depending on the data rate available, the communication channel transmits data between the remote observer location and the local predictor corrector (PC) based controller, only at time instants $\left\{t_{k}\right\}_{k=0}^{\infty}$. It is assumed that this "sampling" of remote data occurs at equally spaced intervals so that the inter-sample time is $t_{k+1}-t_{k}=\sigma \forall k=0,1, \ldots$. Hence the states of (4) are reset to the states estimated by (3) at the sampling instants $\left\{t_{k}\right\}_{k=0}^{\infty}$.

$$
x_{n}\left(t_{k}\right)=\bar{x}\left(t_{k}\right) \text { for all } k=0,1,2, \ldots
$$

The control input is synthesized using the nominal model (4) and (5) according to the following equation:

$$
u(t)=-K x_{n}(t)
$$

where $K \in \Re^{1 \times m}$ is the state feedback gain vector designed based on the nominal system model $G_{n}$.
During time interval $\sigma$, when the reduced order system states are not available from the sensor (i.e. $\mathrm{T}$ is open, see Fig. 1) the system nominal model $G_{n}$ predicts the states. Upon arrival of the next available sample of $\bar{x}(t)$ the states of $G_{n}$ are corrected/reset leading to a switched control strategy.

\section{OVERALl Stability}

Combining equations (2), (4), (6) and (3) the overall system dynamics during the time interval $t \in\left[t_{k}, t_{k+1}\right), t_{k+1}-t_{k}=\sigma$ can be described as:

$$
\left[\begin{array}{c}
\dot{x}_{i} \\
\dot{x}_{n} \\
\dot{\bar{x}}
\end{array}\right]=\left[\begin{array}{ccc}
A_{i} & -B_{i} K & 0 \\
0 & A_{n}-B_{n} K & 0 \\
L C_{i} & -B_{n} K & A_{n}-L C_{n}
\end{array}\right]\left[\begin{array}{c}
x_{i} \\
x_{n} \\
\bar{x}
\end{array}\right]
$$

with the additional condition imposed by (5) at all $t_{k}$. The initial condition $x_{i}(0)$ is usually unknown while the initial conditions for the nominal and the observer states are assumed to be zero $x_{n}(0)=0$ and $\bar{x}(0)=0$.

Following [8], the error $e=\bar{x}-x_{n}$ is defined as the difference between the nominal and estimated (observer) states. Using a linear transformation (7) can be re-written in terms of the error $e(t)$ as follows:

$$
\left[\begin{array}{c}
\dot{x}_{i} \\
\dot{\bar{x}} \\
\dot{e}
\end{array}\right]=\left[\begin{array}{ccc}
A_{i} & -B_{i} K & B_{i} K \\
L C_{i} & A_{n}-L C_{n}-B_{n} K & B_{n} K \\
L C_{i} & -L C_{n} & A_{n}
\end{array}\right]\left[\begin{array}{c}
x_{i} \\
\bar{x} \\
e
\end{array}\right]
$$

It can be proved that the system (8) with periodic resetting is globally, exponentially stable around the solution $\left[\begin{array}{lll}x_{i} & \bar{x} & e\end{array}\right]^{T}=$ $\left[\begin{array}{lll}0 & 0 & 0\end{array}\right]^{T}$ if and only if the eigenvalues of (9) lie inside the unit circle [8].

$$
\Lambda=\left[\begin{array}{lll}
I & 0 & 0 \\
0 & I & 0 \\
0 & 0 & 0
\end{array}\right] e^{\Gamma \sigma}\left[\begin{array}{ccc}
I & 0 & 0 \\
0 & I & 0 \\
0 & 0 & 0
\end{array}\right]
$$

where $\Gamma$ is the overall state matrix in (8). Maximum allowable update interval $\sigma$ i.e. minimum data rate can be obtained from the eigenvalues of (9).

It should be noted here that while (9) characterizes global exponential stability of the linear switched system (8), equivalent stability results characterizing the minimum allowable data rate for general non-linear systems were derived in [10]. However, for practical purposes, the nonlinear model of the power system is quite complex and typically of too high order to be suitable for controller synthesis. Hence for such systems, stability and performance is illustrated through extensive simulations reported in Section VI.

\section{State Trajectories}

The predictor corrector (PC) strategy is based on exploiting the knowledge of nominal system dynamics. Hence, the closed-loop performance is expected to deteriorate as the actual operating condition drifts away from the nominal. It is useful to estimate the deterioration in performance under off-nominal conditions which would depend on the state trajectories of $G_{i}$ during the period between two consecutive feedback samples.

During the inter-sample period $\left[t_{k}, t_{k+1}\right)$ the dynamics of the overall system including the power systems $G_{i}$ and the 
predictor corrector (PC) based controller is expressed by (7). It can be seen that the responses of $x_{i}(t)$ and $x_{n}(t)$ are uncoupled with that of the observer $\bar{x}(t)$. Hence, the left upper block can be considered separately for analysis during $t \in\left[t_{k}, t_{k+1}\right)$. Thus, neglecting observer dynamics without loss of generality, (7) can be rewritten as:

$$
\left[\begin{array}{c}
\dot{x}_{i}(t) \\
\dot{x}_{n}(t)
\end{array}\right]=\left[\begin{array}{cc}
A_{i} & -B_{i} K \\
0 & A_{n}-B_{n} K
\end{array}\right]\left[\begin{array}{c}
x_{i}(t) \\
x_{n}(t)
\end{array}\right]
$$

Note that the state $x_{n}\left(t_{k}\right)$ is reset to the estimated observer state $\bar{x}\left(t_{k}\right)$ according to (5). Thus the initial conditions for (10) are the states at the last available sampling instant $t_{k}$ :

$$
\left[\begin{array}{c}
x_{i}\left(t_{k}\right) \\
x_{n}\left(t_{k}\right)
\end{array}\right]=\left[\begin{array}{l}
x_{k}^{\prime} \\
x_{k}
\end{array}\right]
$$

where

$$
x_{n}\left(t_{k}\right)=\bar{x}\left(t_{k}\right)=x_{k}
$$

Solution of (10) gives:

$$
\left[\begin{array}{l}
x_{i}(t) \\
x_{n}(t)
\end{array}\right]=\exp \left\{\left[\begin{array}{cc}
A_{i} & -B_{i} K \\
0 & A_{n}-B_{n} K
\end{array}\right]\left(t-t_{k}\right)\right\}\left[\begin{array}{l}
x_{k}^{\prime} \\
x_{k}
\end{array}\right]
$$

Equation (12) represents the temporal evolution of system states of the reduced order model and those predicted by $G_{n}$. The state trajectory of $G_{n}$ with initial state $x_{k}$ can be expressed as:

$$
x_{n}(t)=e^{\left(A_{n}-B_{n} K\right)\left(t-t_{k}\right)} x_{k} \text { for } t \in\left[t_{k}, t_{k+1}\right)
$$

Analytical expression for the trajectory of the states of the reduced order linearized power system model is derived as follows. Transforming (12) to Laplace domain we get:

$$
\begin{aligned}
& {\left[\begin{array}{l}
X_{i}(s) \\
X_{n}(s)
\end{array}\right]=\mathcal{L}\left[\exp \left\{\left[\begin{array}{cc}
A_{i} & -B_{i} K \\
0 & A_{n}-B_{n} K
\end{array}\right]\left(t-t_{k}\right)\right\}\right]\left[\begin{array}{c}
x_{k}^{\prime} \\
x_{k}
\end{array}\right]} \\
& =\left[\begin{array}{cc}
\left(s I-A_{i}\right)^{-1} & -\left(s I-A_{i}\right)^{-1} B_{i} K\left(s I-A_{n}+B_{n} K\right)^{-1} \\
0 & \left(s I-\left(A_{n}-B_{n} K\right)\right)^{-1}
\end{array}\right]\left[\begin{array}{c}
x_{k}^{\prime} \\
x_{k} \\
(14)
\end{array}\right]
\end{aligned}
$$

After simplification the expression for $X_{i}(s)$ can be written as:

$$
\begin{aligned}
& X_{i}(s)=\left(s I-A_{n}+B_{n} K\right)^{-1} x_{k}+\left(s I-A_{i}\right)^{-1}\left(x_{k}^{\prime}-x_{k}\right) \\
& +\left(s I-A_{i}\right)^{-1}(\tilde{A}-\tilde{B} K)\left(s I-A_{n}+B_{n} K\right)^{-1} x_{k}
\end{aligned}
$$

Notably $\tilde{A}$ and $\tilde{B}$ represent the deviation of the nominal dynamics embedded in $G_{n}$ from the linearized model of the actual system (corresponding to a particular operating scenario). Thus the state trajectories are given by:

$$
\begin{aligned}
& x_{i}(t)=e^{\left(A_{n}-B_{n} K\right)\left(t-t_{k}\right)} x_{k}+e^{A_{i}\left(t-t_{k}\right)}\left(x_{k}^{\prime}-x_{k}\right) \\
& +\int_{t_{k}}^{t} e^{A_{i}(t-\tau)}(\tilde{A}-\tilde{B} K) e^{\left(A_{n}-B_{n} K\right) \tau} x_{k} d \tau
\end{aligned}
$$

\section{ERror Due to OfF-NOMINAl Dynamics}

The difference between the reduced order linearized system state trajectories and those estimated by the nominal model influences the performance of the PC strategy for an offnominal condition. The error in state trajectories can be expressed as:

$$
\begin{aligned}
& E(t):=x_{i}(t)-x_{n}(t) \\
& =e^{A_{i}\left(t-t_{k}\right)}\left(x_{k}^{\prime}-x_{k}\right)+\int_{t_{k}}^{t} e^{A_{i}(t-\tau)}(\tilde{A}-\tilde{B} K) e^{\left(A_{n}-B_{n} K\right) \tau} x_{k} d \tau
\end{aligned}
$$

The first term in (17) represents the deviation of linearized system state from its asymptotic estimate computed by the observer at $t=t_{k}$. The second term arises due to the difference between actual power system operating scenario and the nominal dynamics. As expected, if both the initial condition error and the model mismatch can be reduced to zero, i.e.

$$
x_{k}^{\prime}=x_{k} ; \quad \tilde{A}=0, \tilde{B}=0
$$

the error $E(t)$ ceases to exist. However, because of changes in operating conditions in practical systems, (18) does not hold good and there is a finite error.

Assuming stable open-loop system there are constants $k_{1}>$ 0 and $\alpha_{1}>0$ such that for any vector $c_{1} \in \Re^{m}$ :

$$
\left\|e^{A_{i} t} c_{1}\right\| \leq k_{1} e^{-\alpha_{1} t}\left\|c_{1}\right\|
$$

Moreover, the closed-loop nominal system is stable and welldamped with the designed controller implying there exists constants $k_{2}>0$ and $\alpha_{2}>0$ such that for any vector $c_{2} \in \Re^{m}$ :

$$
\left\|e^{\left(A_{n}-B_{n} K\right) t} c_{2}\right\| \leq k_{2} e^{-\alpha_{2} t}\left\|c_{2}\right\|
$$

Using (19) and (20) an estimate of the error $E(t)$ can be derived as follows:

$$
\begin{aligned}
& \|E(t)\| \leq\left\|e^{A_{i}\left(t-t_{k}\right)}\right\|\left\|\left(x_{k}^{\prime}-x_{k}\right)\right\| \\
& +\left\|\int_{t_{k}}^{t} e^{A_{i}(t-\tau)}(\tilde{A}-\tilde{B} K) e^{\left(A_{n}-B_{n} K\right) \tau} x_{k} d \tau\right\| \\
& \Rightarrow\|E(t)\| \leq k_{1}\left\|\left(x_{k}^{\prime}-x_{k}\right)\right\| e^{-\alpha_{1}\left(t-t_{k}\right)} \| \\
& +k_{1} k_{2}\|(\tilde{A}-\tilde{B} K)\|\left\|x_{k}\right\| \frac{\left[e^{-\alpha_{2}\left(t-t_{k}\right)}-e^{-\alpha_{1}\left(t-t_{k}\right)}\right]}{\left(\alpha_{1}-\alpha_{2}\right) e^{\alpha_{2} t_{k}}}
\end{aligned}
$$

Equation (21) provides an estimate of the maximum possible error during the inter-sample duration $\left[t_{k}, t_{k+1}\right]$. Assuming $\left(x_{k}^{\prime}-x_{k}\right)=0$, from (21), it can be seen that the upper bound of the intersample error norm is proportional to the mismatch between the nominal and actual model i.e there is a constant $k_{3} \in \mathbb{R}$ such that:

$$
\|E(t)\| \leq k_{3}\|\tilde{A}-\tilde{B} K\|
$$

This relation verifies that the performance of the proposed PC based controller deteriorates in proportion with the model mismatch.

\section{CASE STUDY}

\section{A. Test system}

To illustrate the effectiveness of the predictor corrector (PC) strategy under low feedback data rate situation, case studies were carried out with a 4-machine, 2-area power system shown in Fig. 2 [24]. This test system is simple but representative of 


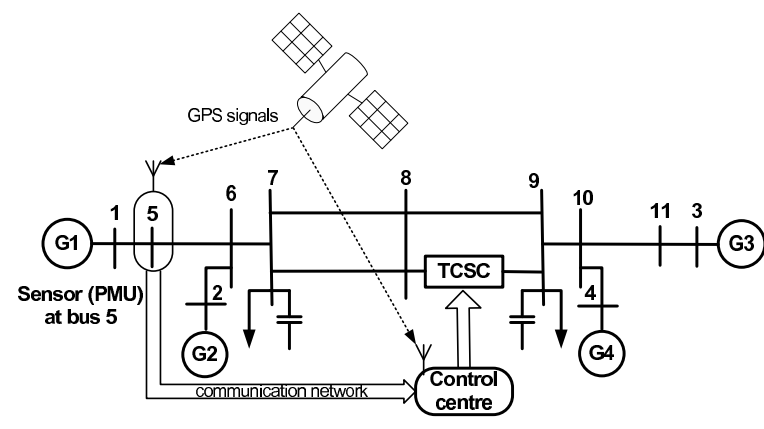

Fig. 2. 4-machine, 2-area test system with a TCSC

typical low frequency electromechanical oscillatory problems encountered in power systems.

Each of the four generators (G1-G4) are represented by a sub-transient model with DC excitation [24]. Under nominal condition, approximately $400 \mathrm{MW}$ power flows from area 1 to area 2 over the two parallel $220 \mathrm{~km}$ tie lines connecting buses 7 and 9 through bus 8 . To control and facilitate this tieline power flow, a thyristor controlled series capacitor (TCSC) [25] is installed to provide $10 \%$ compensation in steady state with a dynamic range of variation from 1 to $50 \%$. Further details of the system can be found in [24]. Linear analysis about nominal operating scenario reveals one poorly damped (about 1\%) electromechanical oscillatory mode with $0.6 \mathrm{~Hz}$ frequency. The objective of this exercise is to improve the damping of this mode by modulating the compensation of the TCSC.

The phase angle of the voltage measured at bus 5 was considered as the feedback signal with bus 11 as the reference bus. A phasor measurement unit (PMU) at bus 5 measures the phase angle of the voltage which is communicated to the distant control centre. Signals from a GPS satellite are used to synchronizes the measurements through precise time-stamp information [18].

The nominal and off-nominal operating scenarios considered for the case study are summarized in Table I.

TABLE I

OPERATING SCENARIOS FOR THE TEST SYSTEM IN FIG. 2

\begin{tabular}{|c||c||c||c|}
\hline No. & Identifier & Tie line power flow & Outage of tie-lines \\
\hline 1 & nominal & $400 \mathrm{MW}$ & none \\
\hline 2 & $8-9$ outage & $400 \mathrm{MW}$ & one between 8 and 9 \\
\hline 3 & $7-8$ outage & $400 \mathrm{MW}$ & one between 7 and 8 \\
\hline 4 & heavy transfer & $800 \mathrm{MW}$ & none \\
\hline
\end{tabular}

\section{B. Control with $C F$ and $P C$ approaches}

For a conventional feedback (CF) controller, the measured signals from the remote sensor - phasor measurement unit (PMU) at bus 5 in this case - is communicated to the controller at the actuator location. Under normal conditions, the communication involves about $20 \mathrm{~ms}$ delay due to PMU sampling rate of $50 \mathrm{~Hz}$. The $\mathrm{CF}$ receives the constant value of the last available sample during the inter-sample interval in the form of a zero-order hold. Here the controller is designed using linear quadratic regulator (LQR) approach [26] based on a 5th order reduced equivalent of the nominal system.
For the predictor corrector (PC) strategy, states of the reduced order system (not the measured outputs) estimated by an observer are communicated over the network. A reduced order system model is used to calculate the control input $(\bar{u}(t))$ required by the observer at the sensor location, see Fig. 1. A 5th order reduced equivalent of the nominal system drives a state feedback controller at the actuator location as described in Section II. In this exercise, balanced truncation [27] was used to obtain the reduced order nominal model of the power system. For large scale power systems subspace based techniques for model reduction could be employed.

\section{Overall stability with PC strategy}

As explained in Section III the overall switched system is stable if the eigenvalues of (9) lie within the unit circle in a z-plane. Stability would dictate the minimum allowable data rate for different operating conditions which could be figured out from (9). The magnitudes of the maximum eigenvalue of (9) for different data rates across varying operating scenarios are shown in Fig. 3.

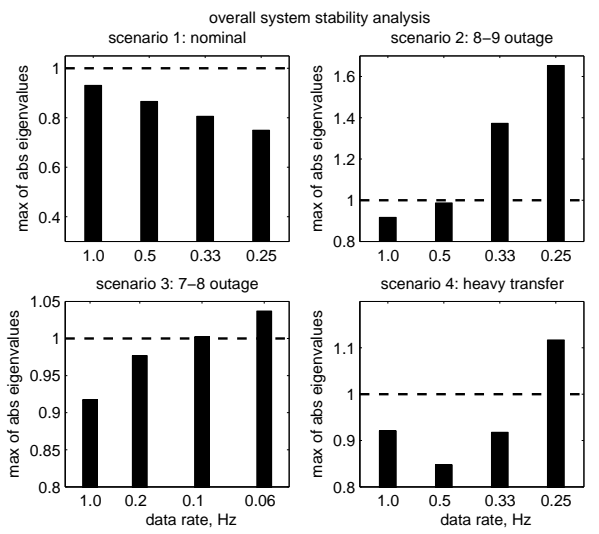

Fig. 3. Magnitudes of maximum eigenvalue of (9) for different data rates across varying operating conditions

Since the PC approach uses knowledge of nominal system dynamics, stability under nominal condition (upper left subplot) is guaranteed with virtually any data rate. However, for 89 outage (upper right subplot) the system becomes unstable for a feedback data rate of $0.33 \mathrm{~Hz}$. Similarly, for 7-8 outage and heavy loading (see Table I) the closed-loop system is unstable below data rates of $0.06 \mathrm{~Hz}$ (lower left subplot) and $0.25 \mathrm{~Hz}$ (lower right subplot), respectively. It should be noted that the eigenvalues of (9) indicates stability only and is not necessarily representative of closed-loop performance [8].

Although (9) characterizes global exponential stability of the linear switched system, the stability of the non-linear power system under switching is not guaranteed [28]. Therefore, non-linear simulations were carried out in Matlab Simulink to validate the stability limits given by (9).

A three phase short circuit at $\mathrm{t}=5.0 \mathrm{~s}$ for $80 \mathrm{~ms}$ ( 5 cycles) near bus 8 was considered as the disturbance. Closed-loop behavior of the system across various scenarios considering different data rates are shown in Fig. 4. For the nominal scenario the closed-loop response is stable even with a data 
rate as low as $0.25 \mathrm{~Hz}$. For scenarios 2, 3 and 4, the closedloop response becomes unstable below data rates of $0.25 \mathrm{~Hz}$, $0.06 \mathrm{~Hz}$ and $0.25 \mathrm{~Hz}$, as obtained from linear analysis and shown in Fig. 3. Thus, the minimum allowable data rates for stability from the linear analysis of the switched system is found to correspond to the nonlinear simulation results.
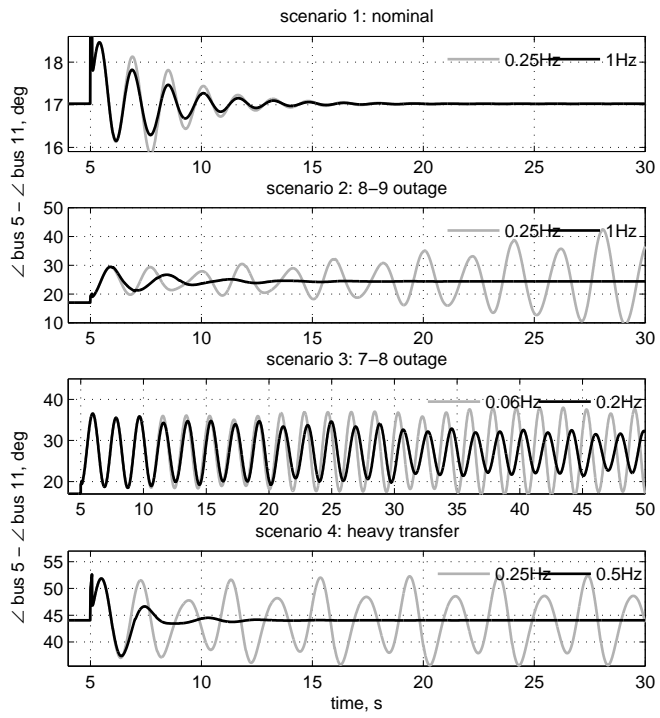

Fig. 4. Closed-loop responses with PC strategy for different feedback data rates across a range of operating conditions

It is to be noted that data rates as low as $0.25 \mathrm{~Hz}$ is not very likely in practice even with shared communication links. Thus PC strategy practically ensures stability as demonstrated here for a range of representative scenarios.

\section{Comparison between $C F$ and PC for low data rates}

Following stability analysis, the closed-loop performance with PC strategy is compared against CF. Before that the
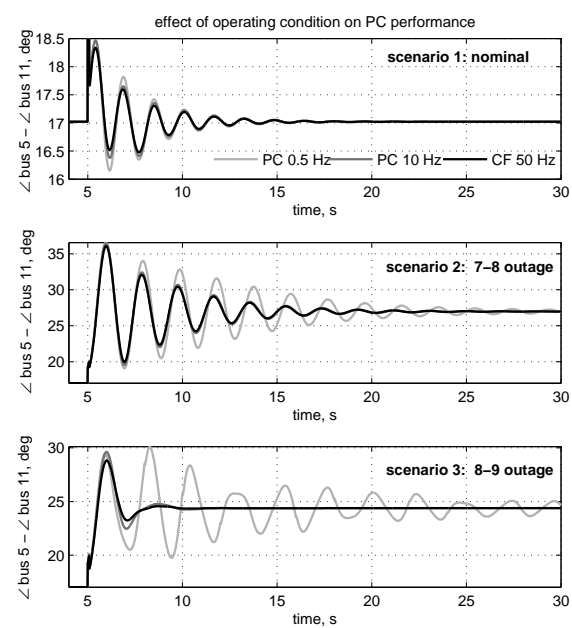

Fig. 5. Effect of change in operating condition on performance with PC

deterioration in closed-loop performance with PC as the scenario drifts away from nominal is illustrated. It is obvious that the difference between the nominal and the actual operating scenario would affect the closed-loop performance as the PC strategy is based on the knowledge of nominal system dynamics. To understand this effect the closed-loop performance with PC using a low data rate $(0.5 \mathrm{~Hz})$ is benchmarked against $\mathrm{CF}$ at nominal data rate of $50 \mathrm{~Hz}$ across different operating conditions. The expected deterioration in performance under off-nominal conditions is evident in Fig. 5.

For the nominal scenario, PC produces almost similar response with different data rates $(0.5 \mathrm{~Hz}, 10 \mathrm{~Hz})$ as $\mathrm{CF}$ does with $50 \mathrm{~Hz}$. However, for off-nominal scenarios (2 and 3 ) the performance of PC gets worse for low data rates. A comparison between closed-loop performance with $\mathrm{PC}$ and $\mathrm{CF}$ is shown in Figs. 6 - 8 for the three off-nominal scenarios (24).
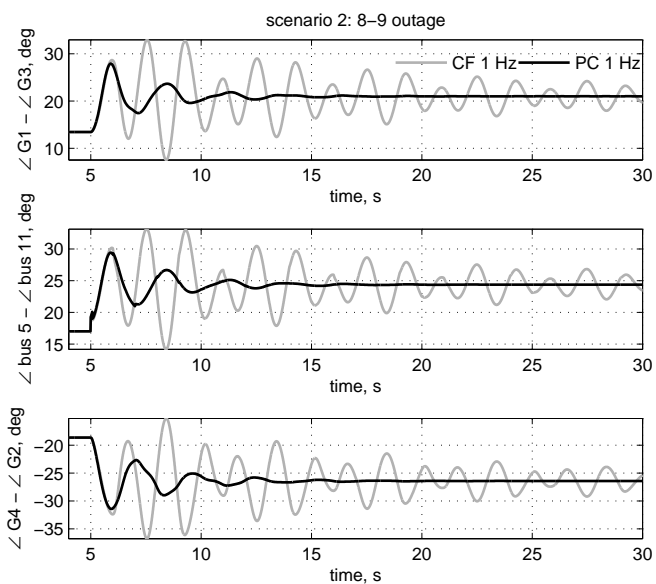

Fig. 6. Comparison between closed-loop performance with $\mathrm{PC}$ and $\mathrm{CF}$ at 1 $\mathrm{Hz}$ data rate for line $8-9$ outage
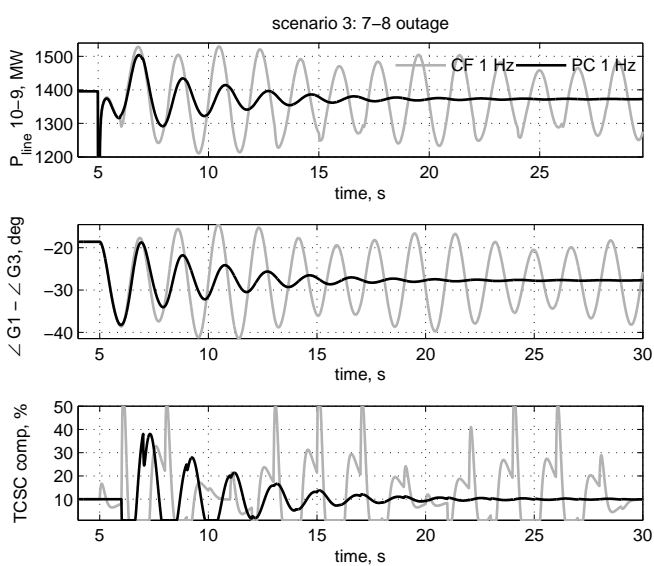

Fig. 7. Comparison between closed-loop performance with $\mathrm{PC}$ and $\mathrm{CF}$ at 1 $\mathrm{Hz}$ data rate for line $7-8$ outage

The same data rate $-1 \mathrm{~Hz}$ - is considered in each case. Along with phase angle difference between generator and other buses and active power flow in lines, dynamic variation of compensation of the TCSC (actuator) is also plotted. From the responses it is clear that for each scenario (see Figs. 6 8) PC produces significantly better closed-loop performance than $\mathrm{CF}$. 

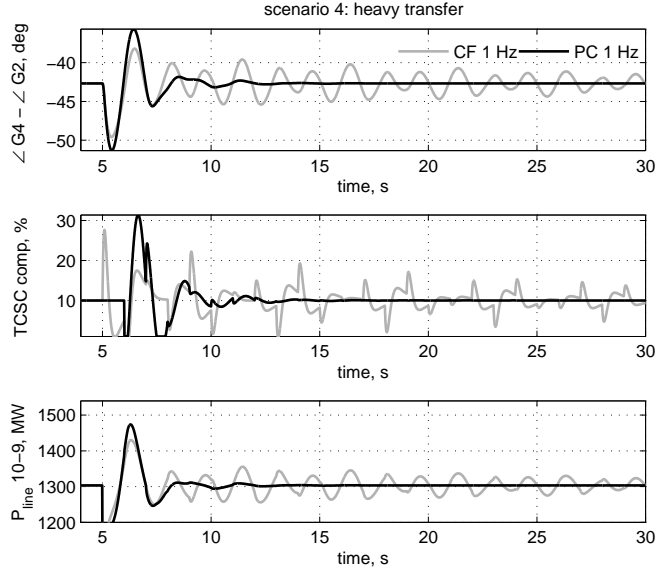

Fig. 8. Comparison between closed-loop performance with $\mathrm{PC}$ and $\mathrm{CF}$ at 1 $\mathrm{Hz}$ data rate for heavy transfer

From the simulation studies, it is clear that even for a data rate of $1 \mathrm{~Hz}$, which is realistically as low as it can get with worst possible communication bandwidth encountered in relevant practical applications, PC strategy not only guarantees closed-loop stability but also produces satisfactory closed-loop responses while the performance with $\mathrm{CF}$ is unacceptable. For higher and more realistic data rates $(2-5 \mathrm{~Hz})$ the difference in performance between PC and CF would be less but enough to justify use of PC during low bandwidth availability.

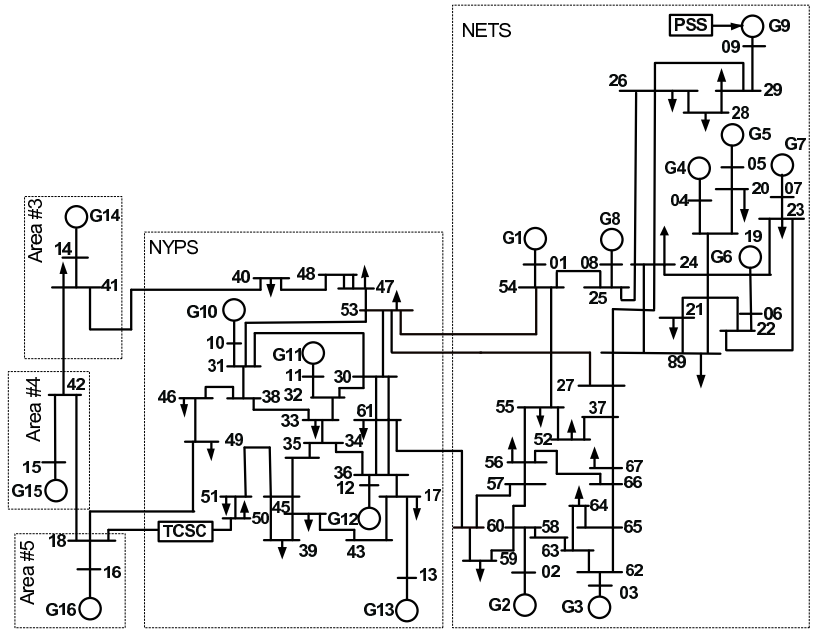

Fig. 9. A 16-machine, 5-area system with a TCSC

\section{E. Case study on a larger power system}

In this section the effectiveness of the proposed approach is illustrated on a relatively larger and realistic 16-machine, 5 -area test system shown in Fig. 9. The details of the system can be found in [29]. The real power flow in line 45-35 was used as the feedback signal to damp power oscillations with a thyristor controlled series capacitor (TCSC) installed on the tie-line connecting the buses 18 and 50. The order of the system is 134 which was reduced to its 10 th order equivalent for controller design. As before a $20 \mathrm{~ms}$ latency (due to 50
$\mathrm{Hz}$ PMU sampling rate) was considered in the communication channel.

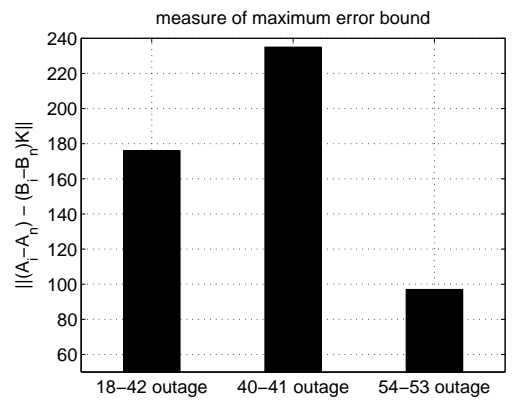

Fig. 10. Maximum error bound of state trajectories in the inter-sample interval for four different operating scenarios

1) Impact of operating condition on PC: Fig. 10 shows the maximum error norm of the state trajectories (22) during the inter-sample interval for three tie-line outage scenarios 18-42, 40-41 and 54-53, see Fig. 9. To demonstrate the correlation between this measure with the actual damping performance of the PC, a low sampling rate of $0.5 \mathrm{~Hz}$ was chosen to allow the state trajectories to evolve freely in the inter-sampling interval and reach its maxima.
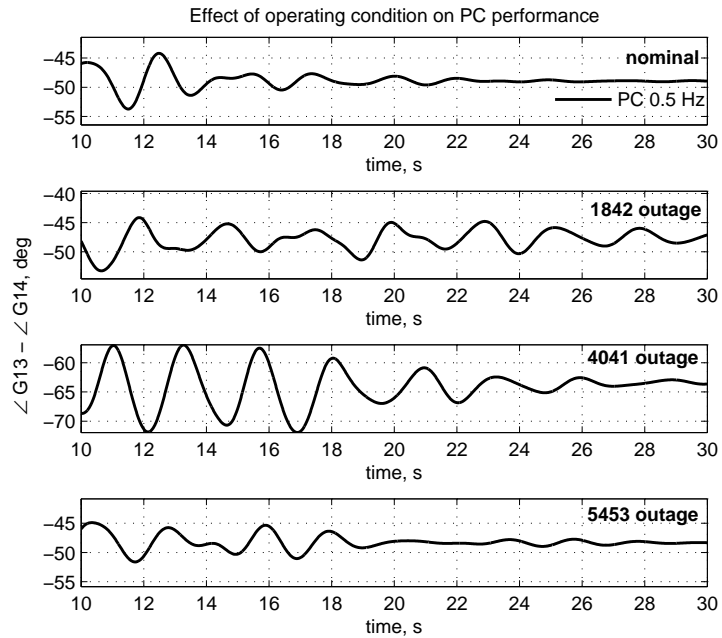

Fig. 11. Impact of change in operating condition on PC performance

Fig. 11 shows the effect of different operating conditions (simulated with a three phase fault followed by three tie-line outages) on the performance of the PC. It can be observed that a qualitative agreement between the maximum error bound (22) shown in Fig. 10 exists in terms of deterioration of dynamic behavior from nominal condition. Note that a similar range for the $y$-axes of the subplots is considered to facilitate a meaningful comparison. Although the fault takes place at $t$ $=5 \mathrm{~s}$, the responses are compared from $\mathrm{t}=10 \mathrm{~s}$ to avoid the immediate effect of the fault in the first two swings.

2) $P C$ vs $C F$ at low data rate: To illustrate that the performance with the $\mathrm{PC}$ is consistently better compared to the $\mathrm{CF}$ for a larger power system, similar contingencies as shown above were simulated. The dynamic behavior of the 
system under these conditions with a $2 \mathrm{~Hz}$ sampling rate is shown in Fig. 12.
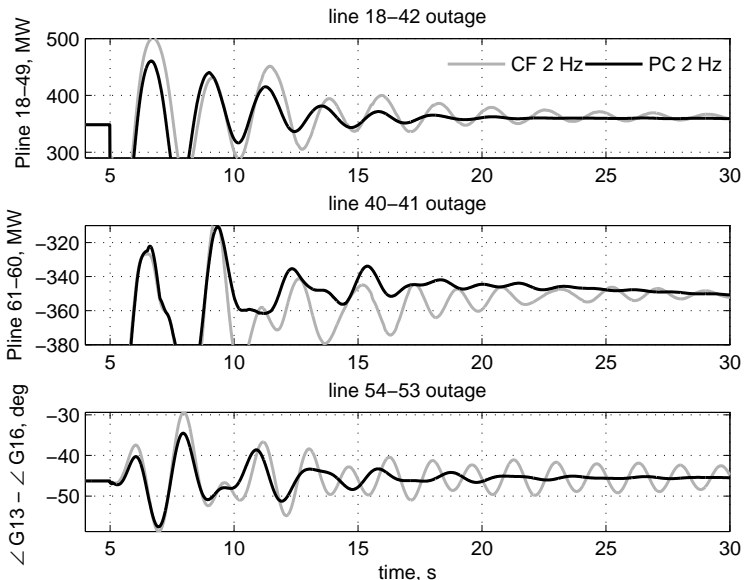

Fig. 12. Comparison of damping performance between $\mathrm{PC}$ and $\mathrm{CF}$ at $2 \mathrm{~Hz}$ data rate for a three phase fault followed by line 18-42, 40-41 and 54-53 outages

\section{CONCLUSION}

In this paper, a predictor corrector (PC) strategy is applied for power oscillation damping control to deal with situations of low feedback data rate (bandwidth) where conventional feedback would suffer. Knowledge of nominal system dynamics is used to approximate (predict) the actual system behavior during intervals when data from remote sensors are not available. Recent samples of the states from a reduced observer at the remote location are used to periodically reset (correct) the nominal dynamics. As expected the closed-loop performance is shown to deteriorate as the actual operating condition drifts away from the nominal dynamics. Nonetheless, significantly better performance compared to conventional feedback is obtained under low bandwidth situations. Simulation results confirm the impact of decreasing data rate on the closed-loop stability of the overall system. It is demonstrated that even for reasonably low data rates the closed-loop stability is usually ensured for a typical power system application confirming the effectiveness of this approach. The deterioration in performance is also quantified in terms of the difference between the nominal and off-nominal dynamics.

\section{REFERENCES}

[1] W. Wing Shing and R. W. Brockett, "Systems with finite communication bandwidth constraints. I. state estimation problems," IEEE Transactions on Automatic Control, vol. 42, no. 9, pp. 1294-1299, 1997.

[2] _ - "Systems with finite communication bandwidth constraints. II stabilization with limited information feedback," IEEE Transactions on Automatic Control, vol. 44, no. 5, pp. 1049-1053, 1999.

[3] O. Beldiman, G. C. Walsh, and L. Bushnell, "Predictors for networked control systems," in Proceedings of the 2000 American Control Conference, vol. 4, 2000, pp. 2347-2351.

[4] G. C. Walsh, Y. Hong, and L. G. Bushnell, "Stability analysis of networked control systems," IEEE Transactions on Control Systems Technology, vol. 10, no. 3, pp. 438-446, 2002.

[5] Y. L. Sun and N. H. El-Farra, "Quasi-decentralized model-based networked control of process systems," Computers \& Chemical Engineering, vol. 32, no. 9, pp. 2016-2029, 2008.
[6] Y. L. Sun, S. Ghantasala, and N. H. El-Farra, "Networked control of distributed energy resources: Application to solid oxide fuel cells," Industrial \& Engineering Chemistry Research, vol. 48, no. 21, pp. 95909602, 2009.

[7] P. V. Zhivoglyadov and R. H. Middleton, "Networked control design for linear systems," Automatica, vol. 39, no. 4, pp. 743-750, 2003.

[8] L. A. Montestruque and P. J. Antsaklis, "On the model-based control of networked systems," Automatica, vol. 39, no. 10, pp. 1837-1843, 2003.

[9] L. A. Montestruque and P. Antsaklis, "Stability of model-based networked control systems with time-varying transmission times," IEEE Transactions on Automatic Control, vol. 49, no. 9, pp. 1562-1572, 2004.

[10] Y. L. Sun and N. H. El-Farra, "Resource-aware quasi-decentralized control of nonlinear plants over communication networks," 2009 American Control Conference, pp. 154-159, 2009.

[11] M. Klein, G. Rogers, and P. Kundur, "A fundamental study of inter-area oscillations in power systems," IEEE Transactions on Power Systems, vol. 6 , no. 3, pp. 914-921, 1991.

[12] I. Kamwa, A. Heniche, G. Trudel, M. Dobrescu, R. Grondin, and D. Lefebvre, "Assessing the technical value of FACTS-based wide-area damping control loops," in proceedings of IEEE Power Engineering Society General Meeting, 2005, 2005, pp. 1734-1743 Vol. 2.

[13] J. Chow, J. Sanchez-Gasca, H. Ren, and S. Wang, "Power system damping controller design-using multiple input signals," IEEE Control Systems Magazine, vol. 20, no. 4, pp. 82-90, 2000.

[14] I. Kamwa, R. Grondin, and Y. Hebert, "Wide-area measurement based stabilizing control of large power systems-a decentralized/hierarchical approach," IEEE Transactions on Power Systems, vol. 16, no. 1, pp. 136-153, 2001.

[15] M. Aboul-Ela, A. Sallam, J. McCalley, and A. Fouad, "Damping controller design for power system oscillations using global signals," IEEE Transactions on Power Systems, vol. 11, no. 2, pp. 767-773, 1996.

[16] J. Y. Cai, H. Zhenyu, J. Hauer, and K. Martin, "Current status and experience of WAMS implementation in north america," in IEEE/PES Transmission and Distribution Conference and Exhibition: Asia and Pacific, 2005, 2005, pp. 1-7.

[17] R. E. Wilson and C. W. Taylor, "Using dynamic simulations to design the wide-area stability and voltage control system (wacs)," in IEEE PES Power Systems Conference and Exposition, 2004, pp. 100-107 vol.1.

[18] C. W. Taylor, D. C. Erickson, K. E. Martin, R. E. Wilson, and V. Venkatasubramanian, "Wacs-wide-area stability and voltage control system: R\&D and online demonstration," Proceedings of the IEEE, vol. 93, no. 5, pp. 892-906, 2005.

[19] G. Heydt, C. Liu, A. Phadke, and V. Vittal, "Solution for the crisis in electric power supply," IEEE Computer Applications in Power, vol. 14, no. 3, pp. 22-30, 2001.

[20] D. Radford, "Spread spectrum data leap through ac power wiring," IEEE Spectrum, vol. 33, no. 11, pp. 48-53, 1996.

[21] D. Schneider, "Is this the moment for broadband over power lines?" IEEE Spectrum, vol. 46, no. 7, pp. 17-17, 2009.

[22] J. W. Stahlhut, T. J. Browne, G. T. Heydt, and V. Vittal, "Latency viewed as a stochastic process and its impact on wide area power system control signals," IEEE Transactions on Power Systems, vol. 23, no. 1, pp. 84-91, 2008.

[23] P. Korba, R. Segundo, A. Paice, B. Berggren, and R. Majumder, "Time delay compensation in power system control," European Union Patent EP08 156 785, filed on May 23, 2008.

[24] P. Kundur, Power system stability and control, ser. The EPRI power system engineering series. New York; London: McGraw-Hill, 1994.

[25] N. G. Hingorani and L. Gyugyi, Understanding FACTS: concepts and technology of flexible AC transmission systems. New York: IEEE Press, 2000.

[26] A. Zolotas, B. Chaudhuri, I. Jaimoukha, and P. Korba, "A study on LQG/LTR control for damping inter-area oscillations in power systems," IEEE Transactions on Control Systems Technology, vol. 15, no. 1, pp. 151-160, 2007.

[27] S. Skogestad and I. Postlethwaite, Multivariable feedback control: analysis and design. Chichester: Wiley, 1996.

[28] M. S. Branicky, "Stability of switched and hybrid systems," in Proceedings of the 33rd IEEE Conference on Decision and Control, 1994, vol. 4 , 1994, pp. 3498-3503 vol.4.

[29] N. R. Chaudhuri, S. Ray, R. Majumder, and B. Chaudhuri, "A new approach to continuous latency compensation with adaptive phasor power oscillation damping controller (pod)," IEEE Transactions on Power Systems, vol. 25, no. 2, pp. 939-946, 2010. 